\title{
The Colored Hofstadter Butterfly for the Honeycomb Lattice
}

\section{Journal Article}

\section{Author(s):}

Agazzi, Andrea; Eckmann, Jean Pierre; Graf, Gian Michele

Publication date:

2014-08

Permanent link:

https://doi.org/10.3929/ethz-b-000087250

Rights / license:

In Copyright - Non-Commercial Use Permitted

Originally published in:

Journal of Statistical Physics 156(3), https://doi.org/10.1007/s10955-014-0992-0 


\title{
The Colored Hofstadter Butterfly for the Honeycomb Lattice
}

\author{
A. Agazzi · J.-P. Eckmann - G.M. Graf
}

Received: 7 March 2014 / Accepted: 29 March 2014 / Published online: 7 May 2014

C Springer Science+Business Media New York 2014

\begin{abstract}
We rely on a recent method for determining edge spectra and we use it to compute the Chern numbers for Hofstadter models on the honeycomb lattice having rational magnetic flux per unit cell. Based on the bulk-edge correspondence, the Chern number $\sigma_{\mathrm{H}}$ is given as the winding number of an eigenvector of a $2 \times 2$ transfer matrix, as a function of the quasi-momentum $k \in(0,2 \pi)$. This method is computationally efficient (of order $\mathcal{O}\left(n^{4}\right)$ in the resolution of the desired image). It also shows that for the honeycomb lattice the solution for $\sigma_{\mathrm{H}}$ for flux $p / q$ in the $r$-th gap conforms with the Diophantine equation $r=\sigma_{\mathrm{H}} \cdot p+s \cdot q$, which determines $\sigma_{\mathrm{H}} \bmod q$. A window such as $\sigma_{\mathrm{H}} \in(-q / 2, q / 2)$, or possibly shifted, provides a natural further condition for $\sigma_{\mathrm{H}}$, which however turns out not to be met. Based on extensive numerical calculations, we conjecture that the solution conforms with the relaxed condition $\sigma_{\mathrm{H}} \in(-q, q)$.
\end{abstract}

Keywords Graphene $\cdot$ Integer quantum Hall effect $\cdot$ Bulk-edge correspondence $\cdot$ Chern number

\section{Introduction}

The spectral diagram of the Hofstadter model for electrons in the 2-dimensional square lattice and in presence of a fractional magnetic flux per unit cell $\Phi / \Phi_{0}=p / q$ has become known as the Hofstadter butterfly [8]. The problem of associating to each spectral gap the corresponding Chern number, representing the integer quantum Hall conductance $\sigma_{\mathrm{H}}$, has been solved in the case of a rectangular lattice potential with perturbative methods (Thouless

\footnotetext{
A. Agazzi · J.-P. Eckmann

Département de Physique Théorique, Université de Genève, 1211 Genève 4, Switzerland

J.-P. Eckmann $(\bowtie)$

Section de Mathématiques, Université de Genève, 1211 Genève 4, Switzerland

e-mail: jean-pierre.eckmann@unige.ch

G. M. Graf

Institut für Theoretische Physik, ETH, 8093 Zürich, Switzerland
} 
et al. [15]), by reduction to a Diophantine equation with a simple window condition, the solution of which is unique. The phase diagram representing the values of $\sigma_{\mathrm{H}}$ as a function of the Fermi energy $E_{\mathrm{F}}$ and of $\Phi / \Phi_{0}$ according to this method has been computed [2] and is known as the colored Hofstadter butterfly. Recently the colored Hofstadter butterfly has also been calculated for the triangular lattice [3].

Using methods from [1] we present results for the analogous problem on the honeycomb lattice, which is in particular the lattice structure of graphene. The Hofstadter Hamiltonian has been considered for the honeycomb lattice potential [9], and the corresponding spectral diagram can be calculated [12]. Furthermore, the analysis for the labeling of the different phases (gaps) of the spectrum can be generalized to the honeycomb lattice, leading to exactly the same Diophantine equation. However, the constraints on its solutions are not the same as in the rectangular case, and a simple algebraic condition for the determination of the Chern numbers of this problem is still lacking.

We circumvent this problem by computing the Chern numbers of the diagram using a different approach: The bulk-edge correspondence $[1,6]$. By computing the winding number of the edge eigenstates along a loop in the Brillouin zone [1] it is possible to assign to each gap its Chern number $\sigma_{\mathrm{H}}$. The fact that the solution so obtained satisfies the Diophantine equation is evidence to the reliability of the method.

\section{The Natural Window Condition and Its Exceptions}

The honeycomb lattice can be viewed as two interpenetrating triangular lattices (labeled by letters $A$ and $B$ ) as displayed in Fig. 1. As a consequence, the wave function of an electron in the tight binding approximation on this bipartite lattice can be written as the spinor

$$
\psi_{m, n}=\left(\begin{array}{l}
\psi_{m, n}^{A} \\
\psi_{m, n}^{B}
\end{array}\right) \in \mathbb{C}^{2}, \quad \psi=\left(\psi_{m, n}\right)_{m, n} \in \mathcal{H}=\ell^{2}\left(\mathbb{Z}^{2} ; \mathbb{C}^{2}\right),
$$

where $(m, n)$ label the sites on the Bravais sublattice $A$ (or $B$ ).

We define the nearest-neighbor $(N N)$ magnetic hopping operators $T_{i}: \mathcal{H} \rightarrow \mathcal{H},(i=$ $1,2,3)$ by

$$
\begin{aligned}
& \left(T_{1} \psi\right)_{m, n}=\left(\begin{array}{l}
\psi_{m, n}^{B} \\
\psi_{m, n}^{A}
\end{array}\right), \\
& \left(T_{2} \psi\right)_{m, n}=\left(\begin{array}{l}
\psi_{m+1, n}^{B} \\
\psi_{m-1, n}^{A}
\end{array}\right), \\
& \left(T_{3} \psi\right)_{m, n}=\left(\begin{array}{cc}
e^{-2 \pi i \Phi m} & \psi_{m, n+1}^{B} \\
e^{2 \pi i \Phi m} & \psi_{m, n-1}^{A}
\end{array}\right),
\end{aligned}
$$

and this fixes our choice of gauge. The Hofstadter model on the honeycomb lattice is an isotropic $N N$ hopping Hamiltonian which can be written as

$$
H=\sum_{j=1}^{3} T_{j} .
$$




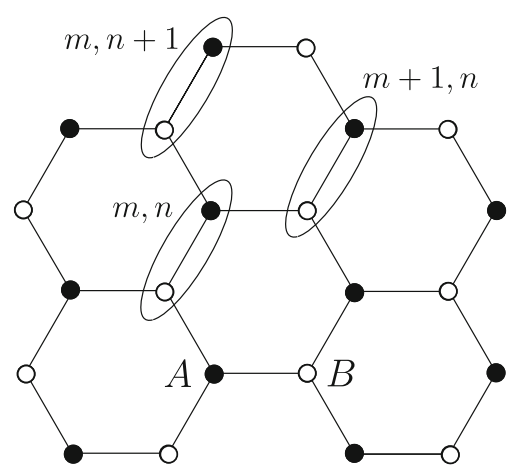

Fig. 1 Coordinate system on the honeycomb lattice structure

Because of the translation invariance of $H$ in the $n$-direction, a Bloch decomposition in that direction can be performed:

$$
\psi_{m, n}=\int_{0}^{2 \pi} \mathrm{d} k \psi_{m}(k) e^{i k n} .
$$

This transformation fibers $H$, which can now be written as

$$
(H(k) \psi(k))_{m}=A^{*}(k) \psi_{m-1}(k)+V_{m}(k) \psi_{m}(k)+A(k) \psi_{m+1}(k),
$$

where

$$
A(k)=\left(\begin{array}{ll}
0 & 1 \\
0 & 0
\end{array}\right) \quad \text { and } \quad V_{m}(k)=\left(\begin{array}{cc}
0 & 1+e^{i(k-2 \pi \Phi m)} \\
1+e^{-i(k-2 \pi \Phi m)} & 0
\end{array}\right) .
$$

\subsection{The Diophantine Equation}

The magnetic field reduces the translation symmetry of the Hamiltonian. In the case of rational fluxes $\Phi=p / q$, the gauge underlying 2.1 increases the translation invariance period of the Hamiltonian in the $m$-direction from 1 to $q$ lattice units. (In the $n$-direction the translation invariance is not broken.) A Bloch decomposition in the $m$-direction can thus be performed and, by plotting the eigenvalues of the Hamiltonian fiber by fiber, the (black and white) Hofstadter butterfly of the honeycomb lattice is drawn [12]. Moreover, by performing an analysis similar to the one carried out in [15] or by using that $H$ commutes with magnetic translations, one can see that the Hall conductivity in the $r$-th gap satisfies the Diophantine equation $[3,5]$

$$
r=\sigma_{\mathrm{H}} \cdot p+s \cdot q .
$$

This is the same equation as in the square lattice case. It clearly determines $\sigma_{\mathrm{H}}$ up to a mod $q$ ambiguity. The natural "window condition" [15],

$$
\sigma_{\mathrm{H}} \in(-q / 2, q / 2)
$$

resolves this ambiguity in the square lattice case. Unfortunately, 2.5 neither holds for the triangular lattice [3] nor for the honeycomb lattice, which we study here. We illustrate this in Table 1 and Fig. 2 for the honeycomb lattice.

This is evidence to the fact that there are topological obstructions to the adiabatic deformation of one lattice into the other, i.e., spectral gaps are in general closing when the lattice is 
Table 1 Failure of the natural window condition 2.5 for $p / q=1 / 5,1 / 6,1 / 7,2 / 5$

\begin{tabular}{|c|c|}
\hline $1 / 5$ & $1,2, \not 2 \rightarrow 3,-1,0,1, \not 2 \rightarrow-3,-2,-1$ \\
\hline $1 / 6$ & $1,2,3,-2 \rightarrow 4,-1,0,1, \not 2 \rightarrow-4,-3,-2,-1$ \\
\hline $1 / 7$ & $1,2,3,-3 \rightarrow 4,-2 \rightarrow 5,-1,0,1, \not 2 \rightarrow-5, \not 2 \rightarrow-4,-3,-2,-1$ \\
\hline $2 / 5$ & $\chi 2 \rightarrow 3,1,-1,2,0,-2,1,-1, \not 2 \rightarrow-3$ \\
\hline
\end{tabular}

There are $2 q-1$ gaps, and in some of them the natural window condition does not predict the correct value of $\sigma_{\mathrm{H}}$. For example, the first wrong prediction is -2 , but the correct value of $\sigma_{\mathrm{H}}$ is 3
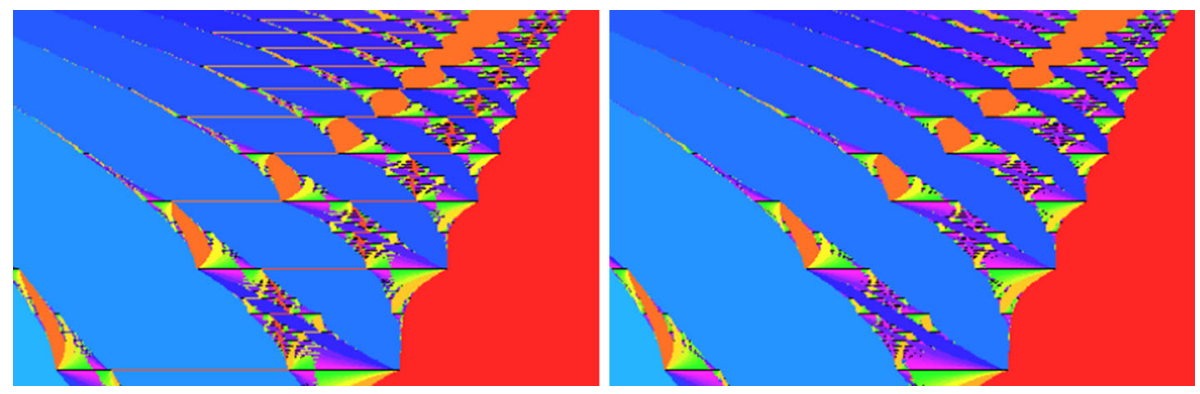

Fig. 2 Comparison of the Chern numbers obtained from the natural window condition 2.5 (left) and the correct ones (right, using the bulk-edge correspondence) for the honeycomb lattice. Note the errors (orange horizontal lines on the left). The horizontal axis represents the energy, the vertical axis the applied magnetic field. Different colors in the figure represent different values of the Chern numbers as shown in Fig. 5. Black lines indicate spectral bands

deformed as a consequence of the von Neumann-Wigner theorem. We expect such problems to occur in other lattices as well.

A way around this problem would be to compute $\sigma_{\mathrm{H}}$ using the Streda formula [14]. This, however, leads to a computational cost which grows exponentially with $q$, which represents an upper bound to the number of resolvable pixels in the $y$-direction and limits in turn the resolution of the output image. Several other attempts towards this objective have been made $[3,7,10,13]$ but no conditions have been found yet that are both general and simple to apply. As a result the computation of the colored Hofstadter butterfly still requires some effort. Some approaches $[7,13]$ make use of bulk-edge correspondence. They detect edge states by finite volume diagonalization which is a method that differs from what we propose here.

\section{The Chern Number Through Bulk-Edge Correspondence}

Instead of considering a bulk honeycomb lattice (infinite in both spatial dimensions) we shall consider one which is infinite in one spatial direction and semi-infinite in the other: An edge honeycomb lattice. The edge profile is assumed to be of zigzag-type, i.e., as the left edge in Fig. 1. As a consequence, instead of a 2-dimensional periodic Hamiltonian $H$, we consider a Hamiltonian $\widehat{H}$ whose action is restricted to the half-space Hilbert space $\widehat{\mathcal{H}}=\ell^{2}\left(\mathbb{N} \times \mathbb{Z} ; \mathbb{C}^{2}\right)$ by means of a Dirichlet boundary condition. Still, $\widehat{H}$ can be fibered by Bloch decomposition in the unbroken symmetry direction, with fibers $\widehat{H}(k)$. Since $H(k)=\widehat{H}(k) \oplus \widehat{H}(k) \oplus R(k)$ with $R(k)$ being a finite rank perturbation, $H(k)$ and $\widehat{H}(k)$ share the same essential spectrum: 


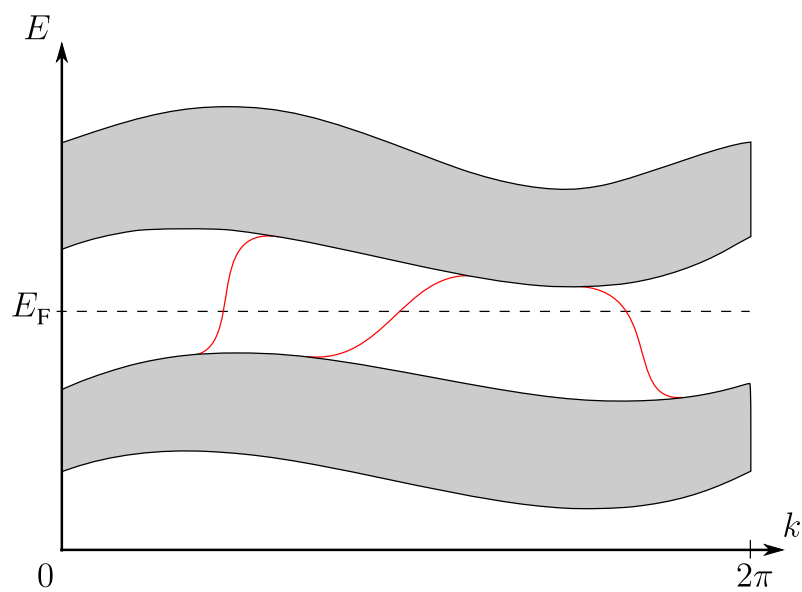

Fig. 3 Intersections of the edge-spectrum (red solid lines) with a fixed level of the Fermi energy $E_{\mathrm{F}}$ (dashed line). There are 2 positive intersections and a negative one

$$
\sigma_{\mathrm{ess}}(\widehat{H}(k))=\sigma_{\mathrm{ess}}(H(k)) .
$$

Hence, the two spectra differ at most by a discrete spectrum in the band gaps of $H(k)$. As a function of $k$, the discrete eigenvalues of $\widehat{H}(k)$ give rise to lines as shown in Fig. 3 . Collectively they form the edge spectrum. By bulk-edge correspondence [6], the (signed) number of such eigenvalues crossing a fixed energy in a spectral gap of $H$ equals the Chern number of that gap. Physically, that energy is the Fermi energy $E_{\mathrm{F}}$ and that number the Hall conductance $\sigma_{\mathrm{H}}$ (in units of $\left.e^{2} / h\right)$.

\subsection{Detection of Edge States}

The task of finding the edge spectrum at an energy $E$ in a gap of the bulk spectrum can be solved based on methods developed in $[1,11]$. For the convenience of the reader we adapt the proofs to the present, simpler setting. The Hamiltonian $\widehat{H}$ is 1 -periodic in the $n$-direction and $q$-periodic in the $m$-direction (in the half-space). In analogy to 2.3, the Schrödinger equation for $\widehat{H}(k)$ can be written as

$$
\left(\begin{array}{l}
a_{m} \psi_{m}^{B}(k)+\psi_{m+1}^{B}(k) \\
\bar{a}_{m} \psi_{m}^{A}(k)+\psi_{m-1}^{A}(k)
\end{array}\right)=E\left(\begin{array}{l}
\psi_{m}^{A}(k) \\
\psi_{m}^{B}(k)
\end{array}\right)
$$

with $a_{m}=a_{m}(k)=e^{i k-2 \pi i m p / q}+1$. Solving with respect to $\psi_{m}^{A}, \psi_{m+1}^{B}$ we obtain, analogously to $[4,7]$,

$$
\left(\begin{array}{c}
\psi_{m+1}^{B}(k) \\
\psi_{m}^{A}(k)
\end{array}\right)=\mathcal{T}_{m}^{E}(k)\left(\begin{array}{c}
\psi_{m}^{B}(k) \\
\psi_{m-1}^{A}(k)
\end{array}\right)
$$

with

$$
\mathcal{T}_{m}^{E}(k)=\frac{1}{\bar{a}_{m}}\left(\begin{array}{cc}
E^{2}-\left|a_{m}\right|^{2}-E \\
E & -1
\end{array}\right) .
$$


Combining these matrices we define the transfer operator over a period of $q$ steps (in the $m$-direction) by

$$
\mathcal{T}^{E}(k)=\prod_{m=1}^{q} \mathcal{T}_{m}^{E}(k)=\mathcal{T}_{q}^{E}(k) \cdots \mathcal{T}_{1}^{E}(k) .
$$

With this definition we have

$$
\left(\begin{array}{c}
\psi_{m+q}^{B}(k) \\
\psi_{m+q-1}^{A}(k)
\end{array}\right)=\mathcal{T}^{E}(k)\left(\begin{array}{c}
\psi_{m}^{B}(k) \\
\psi_{m-1}^{A}(k)
\end{array}\right) .
$$

Let $E$ be an energy in the gap, so that $\mathcal{T}^{E}(k)$ is hyperbolic, in the sense that its eigenvalues satisfy $|\lambda| \neq 1$. An edge state of energy $E$ is a solution of 3.1 and thus of 3.4 that vanishes at the boundary $\left(\psi_{-1}^{A}(k)=0\right)$ and decays for $m \rightarrow \infty$. It exists iff the contracting eigenvector $\Omega(k)=\left(\psi_{0}^{B}(k), \psi_{-1}^{A}(k)\right)$ satisfies the boundary condition, i.e., iff

$$
\Omega(k) \sim\left(\begin{array}{l}
1 \\
0
\end{array}\right),
$$

where $\sim$ stands for proportionality. Edge state energies occur as branches $E(k)$ depending continuously (in fact, analytically) on $k$.

Lemma 1 Let $E$ be a fixed energy in a gap of the Hamiltonian 2.2. The count $N$ of the (signed) crossings of $E$ by eigenvalues of $\widehat{H}(k)$ is half the count of the (signed) number of times condition 3.5 is traversed as the eigenvector winds with $k$.

Proof For the first count, $N$, the crossing is counted positively for decreasing eigenvalue branches, $E^{\prime}(k)<0 .^{1}$

To study the second count, we first note that $\mathcal{T}_{m}=\mathcal{T}_{m}^{E}(k)$ is symplectic (for real energies $E$ ) in the sense that

$$
\mathcal{T}_{m}^{*} \mathcal{J} \mathcal{T}_{m}=\mathcal{J}
$$

with respect to the bilinear form given by

$$
\mathcal{J}=\left(\begin{array}{cc}
0 & -1 \\
1 & 0
\end{array}\right)=-\mathcal{J}^{*}
$$

Therefore, by 3.3 , we see that $\mathcal{T}=\mathcal{T}^{E}(k)$ is symplectic as well:

$$
\mathcal{T}^{*} \mathcal{J} \mathcal{T}=\mathcal{J}
$$

This is physically interpreted as current conservation.

We next observe that the matrix $\mathcal{T}^{E}(k)$ is real up to a factor, since the $\mathcal{T}_{m}^{E}(k)$ in 3.2 are real up to a factor. Being $\mathcal{T}$ symplectic we have $|\operatorname{det} \mathcal{T}|=1$ and the contracting eigenvalue is simple. Its eigenvector can thus be chosen to be real, $\Omega(k)=(a(k), b(k)) \in \mathbb{R}^{2}$, which makes the notion of winding clear. Note that 3.5 will hold twice per full turn of $\Omega(k)$. Therefore, we get the identity

$$
N=\int_{0}^{2 \pi} \frac{\mathrm{d} k}{2 \pi i} \frac{\partial}{\partial k} \log \left(\frac{a(k)+i b(k)}{a(k)-i b(k)}\right) .
$$

1 This seemingly unnatural prescription reflects the negative orientation of the edge, which has its outward normal pointing left. 
In view of the explanations leading to 3.5 , we need to show that the relative signs of the two counts are always the same. To this end we reinstate the dependence on $E$ in $\Omega(E, k)$ and make the vector locally unique by imposing $a(E, k)=1$. Then $b(E(k), k)=0$, and differentiating we get

$$
\frac{\partial b}{\partial E} E^{\prime}(k)+\frac{\partial b}{\partial k}=0 .
$$

The claim will now follow immediately from

$$
\frac{\partial b}{\partial E}>0
$$

at crossing points, which is proven below. In the following $k$ is fixed but we also suppress the dependency on $E$ from the notation. In addition to 3.7, explicit calculation shows that

$$
\mathcal{T}_{m}^{*} \mathcal{J} \frac{\mathrm{d} \mathcal{T}_{m}}{\mathrm{~d} E}=\frac{1}{\left|a_{m}\right|^{2}}\left(\begin{array}{cc}
E^{2}+\left|a_{m}\right|^{2} & -E \\
-E & 1
\end{array}\right)>0 .
$$

Using $\mathcal{T}_{m}^{*} \mathcal{J} \mathcal{T}_{m}=\mathcal{J}$ and

$$
\frac{\mathrm{d} \mathcal{T}}{\mathrm{d} E}=\sum_{m=1}^{q} \mathcal{T}_{q} \cdots \frac{\mathrm{d} \mathcal{T}_{m}}{\mathrm{~d} E} \cdots \mathcal{T}_{1}
$$

we conclude from 3.10 that

$$
\mathcal{T}^{*} \mathcal{J} \frac{\mathrm{d} \mathcal{T}}{\mathrm{d} E}>0
$$

From the definition of $\Omega$, we find

$$
\Omega^{*} \mathcal{J} \frac{\mathrm{d} \Omega}{\mathrm{d} E}=\bar{\psi}_{-1}^{A} \frac{\mathrm{d} \psi_{0}^{B}}{\mathrm{~d} E}-\bar{\psi}_{0}^{B} \frac{\mathrm{d} \psi_{-1}^{A}}{\mathrm{~d} E}=-\frac{\mathrm{d} b}{\mathrm{~d} E},
$$

where the last equality holds at crossing points. We next differentiate $(\mathcal{T}-\lambda) \Omega=0$, where $\lambda$ is the contracting eigenvalue $(|\lambda|<1)$, and obtain

$$
\left(\frac{\mathrm{d} \mathcal{T}}{\mathrm{d} E}-\frac{\mathrm{d} \lambda}{\mathrm{d} E}\right) \Omega+(\mathcal{T}-\lambda) \frac{\mathrm{d} \Omega}{\mathrm{d} E}=0 .
$$

Finally, we multiply the last equality from the left by $\Omega^{*} \mathcal{T}^{*} \mathcal{J}=\bar{\lambda} \Omega^{*} \mathcal{J}$, and using (by the reality of $\Omega) \Omega^{*} \mathcal{J} \Omega=0$, we get

$$
\Omega^{*} \mathcal{T}^{*} \mathcal{J} \frac{\mathrm{d} \mathcal{T}}{\mathrm{d} E} \Omega+\left(1-|\lambda|^{2}\right) \Omega^{*} \mathcal{J} \frac{\mathrm{d} \Omega}{\mathrm{d} E}=0,
$$

where we used 3.7. In view of 3.11 and of 3.12 we conclude $\mathrm{d} b / \mathrm{d} E>0$.

As shown in the Appendix, points $k$ where $a_{m}=0$ do not invalidate the above argument.

\subsection{Numerical Implementation}

We compute the Chern number in each of the spectral gaps of the Hamiltonian using the r.h.s. of 3.8. This involves finding the eigenvectors of $\mathcal{T}^{E}(k)$ for $E$ in a gap, and for sufficiently many $k$ so that the variation of the phase $\theta(k)$ given by the logarithm in 3.8 can be computed with confidence. The issue here is that one must be able to resolve the continuity of $\theta(k)$ when $\partial \theta / \partial k \gg 1$ as shown in Fig. 4. 


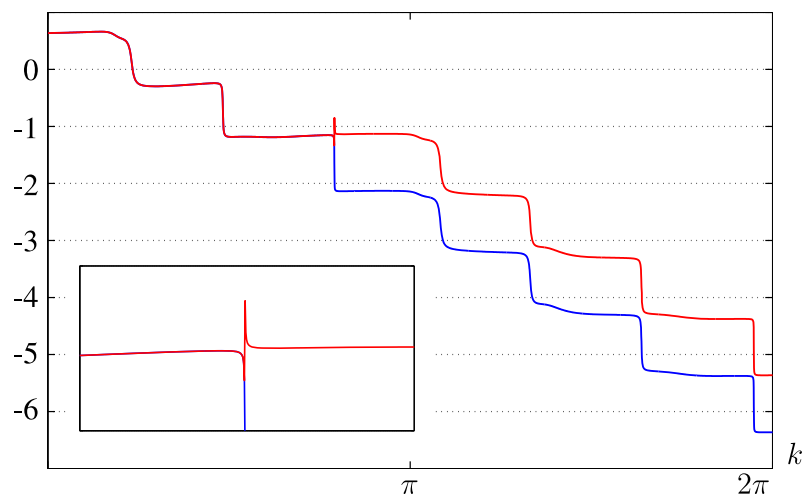

Fig. 4 Evolution of the phase $\theta(k) / 2 \pi$ (on the $y$-axis) as a function of $k \in[0,2 \pi$ ) (on the $x$-axis) for values of $p / q=8 / 19, r=1$. The total winding number of this function is equal to the Chern number associated to the gap. A too low discretization of the interval (red curve, $200 \cdot q$ ) misses a phase turn as shown in the inset; a higher discretization (blue curve, $300 \cdot q$ ) resolves the problem.

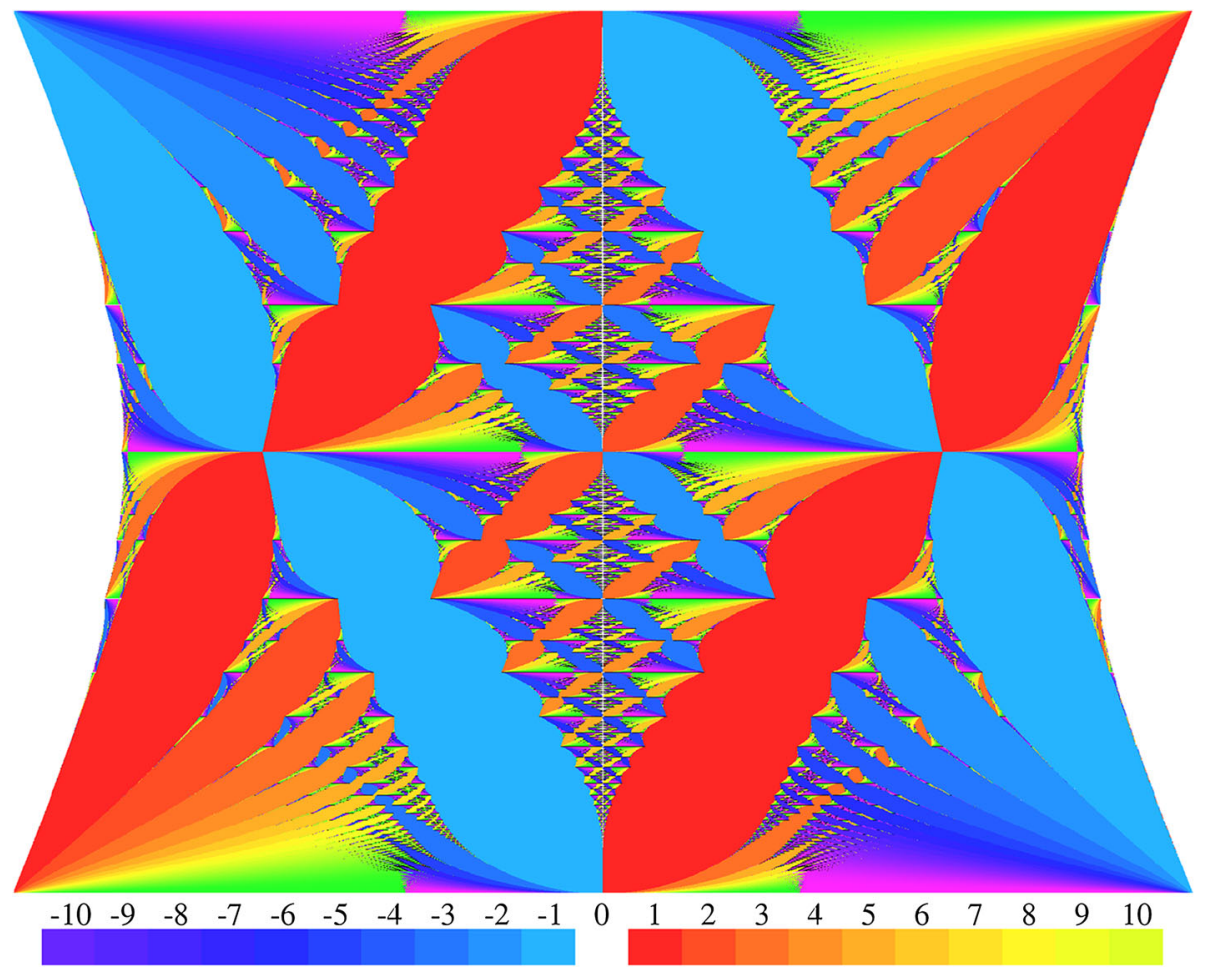

Fig. 5 The colored Hofstadter butterfly for the honeycomb lattice, as obtained by the method of this paper. The vertical axis is the magnetic flux per unit cell $\Phi$ ranging from 0 to 1 . The horizontal axis is the Fermi energy ranging from -3 to 3 . The colors represent the Chern numbers. The resolution of this figure is $1920 \times 1440$ and the maximal value of $q$ is $q_{\max }=720$. Black lines correspond to spectral bands

We compute the Chern number for each gap $\left(e_{1}, e_{2}\right)$ numerically, in the center of the gap. We get some integer $\sigma_{\mathrm{H}}=\sigma_{\mathrm{H}}\left(\left(e_{1}+e_{2}\right) / 2, p, q\right)$ that should be among the solutions of the Diophantine equation 


$$
r=\sigma_{\mathrm{H}} \cdot p+s \cdot q
$$

If it indeed is, we are confident that we have found the correct Chern number. If it is not, we proceed as follows: If it is close to one of the solutions of 3.13, we take that as the solution. Here, we define close as $\left|\sigma_{\mathrm{H}}-\sigma_{*}\right| / q<0.1$, where $\sigma_{*}$ is a solution of 3.13. If it is not, the Chern number in that gap remains undecided. This leads to Fig. 5.

Remark - The computational cost depends on the denominator $q$ and is of the order $\mathcal{O}\left(q^{2} / \Delta k\right)=\mathcal{O}\left(q^{3}\right)$, where $\Delta k$ is the discretization in $k$ which we take as $\Delta k=$ $2 \pi /(200 q)$. Going over all possible fractions $p / q$ for fixed $q$ leads to a cost of at most $\mathcal{O}\left(q^{4}\right)$.

- For each horizontal row of pixels (which represents a small interval of applied magnetic field) we choose $p / q$ in this interval with the smallest possible $q$. The (hardly visible) black horizontal lines represent the spectrum for this choice of $p / q$.

- While the algorithm can in principle always result in a solution of 3.13, there is a limitation to its success. Whenever an eigenvalue of the matrix $\mathcal{T}^{E}(k)$ comes close to $|\lambda|=0$ it contributes significantly to the winding number within a small interval $\Delta k$. This may require a discretization that is finer than reasonably doable.

- The statistics of Fig. 5 are as follows: Of the $\mathcal{O}\left(10^{6}\right)$ colored pixels on the figure, $99.8 \%$ satisfy $3.13,0.1 \%$ are merely close to the correct Chern number, and another $0.1 \%$ are undecided. The smallest gap that can be represented at this resolution is about $e_{2}-e_{1}=$ 0.037 wide. We have no rule to predict which $p$ or $q$ are most likely to lead to difficulties.

- Although 3.13 allows in principle arbitrarily large Chern numbers, we conjecture, based on our calculations, that the Chern numbers lie in $(-q, q)$.

Lossless versions of Figs. 2 and 5 can be obtained from the authors.

Acknowledgments We thank Y. Avron for discussions and for raising the problem addressed in this paper and D. Vvedensky for the co-supervision of the project. The research of AA and JPE was supported in part by an ERC Advanced Grant and that of GMG by the Swiss National Science Foundation.

\section{Appendix}

At values $k$ where $a_{m}=0$ the Schrödinger equation cannot be solved by transfer matrices, and we need a slight modification of the argument. If $a_{m}=0$ then

$$
\bar{a}_{m} \mathcal{T}_{m}=\left(\begin{array}{c}
E^{2}-E \\
E-1
\end{array}\right)=\left(\begin{array}{c}
E \\
1
\end{array}\right)(E,-1)
$$

which has rank 1 . Instead of studying $\mathcal{T}=\mathcal{T}_{q} \cdots \mathcal{T}_{1}$ we study the operator $\widetilde{\mathcal{T}}=\mathcal{T}_{m} \widetilde{\mathcal{T}}_{m}$ which is similar to $\mathcal{T}$, with

$$
\widetilde{\mathcal{T}}_{m}=\mathcal{T}_{m+q-1} \cdots \mathcal{T}_{m+1}
$$

Because of 3.14, one eigenvalue of $\bar{a}_{m} \widetilde{\mathcal{T}}$ is always 0 . The eigenvector of the other eigenvalue of $\bar{a}_{m} \widetilde{\mathcal{T}}$ must be proportional to $(E, 1)^{\mathrm{T}}$, and the eigenvalue is then given by

$$
\bar{a}_{m} \mathcal{T}_{m} \widetilde{\mathcal{T}}_{m}\left(\begin{array}{c}
E \\
1
\end{array}\right)=\left(\begin{array}{c}
E \\
1
\end{array}\right)(E,-1) \widetilde{\mathcal{T}}_{m}\left(\begin{array}{c}
E \\
1
\end{array}\right)=\lambda\left(\begin{array}{c}
E \\
1
\end{array}\right) .
$$


We thus find a second eigenvalue $\lambda \neq 0$ (and in particular no double 0 eigenvalue, i.e., a well-defined and continuous eigenvector $\Omega(k)$ ) unless

$$
(E,-1) \widetilde{\mathcal{T}}_{m}\left(\begin{array}{c}
E \\
1
\end{array}\right)=0 .
$$

The next lemma shows that this never happens, since we are considering $E \notin \sigma(H(k))$.

Lemma 2 If 3.16 holds then $E \in \sigma(H(k))$.

Proof First note $\widetilde{\mathcal{T}}_{m}$ is not enough to find a solution of the Schrödinger equation. However, in the case $a_{m}=0,3.1$ reduces to

$$
\left(\begin{array}{c}
\psi_{m+1}^{B}(k) \\
\psi_{m}^{A}(k)
\end{array}\right) \sim\left(\begin{array}{c}
E \\
1
\end{array}\right), \quad\left(\begin{array}{c}
\psi_{m+q}^{B}(k) \\
\psi_{m+q-1}^{A}(k)
\end{array}\right) \sim\left(\begin{array}{c}
1 \\
E
\end{array}\right) .
$$

Therefore, the condition $a_{m}=0$ and 3.16 imply that there is a solution of $H(k) \psi=E \psi$ which is compactly supported on and between sites $(m, B),(m+q, A)$, or on any interval shifted by multiples of $q$. (Each such state is normalizable.) Thus, $E \in \sigma(H(k)$ ).

\section{References}

1. Avila, J.C., Schulz-Baldes, H., Villegas-Blas, C.: Topological invariants of edge states for periodic twodimensional models. Math. Phys. Anal. Geom. 16, 137-170 (2013)

2. Avron, J.E.: Colored Hofstadter butterflies. In: Multiscale Methods in Quantum Mechanics, Trends Math, pp. 11-22. Birkhäuser, Boston (2004)

3. Avron, J.E., Kenneth, O., Yehoshua, G.: A numerical study of the window condition for Chern numbers of Hofstadter butterflies. http://arxiv.org/abs/1308.3334

4. Bernevig, B., Hughes, T.L., Zhang, S.-C., Chen, H.-D., Wu, C.: Band collapse and the quantum Hall effect in graphene. Int. J. Mod. Phys. B 20, 3257-3278 (2006)

5. Dana, I., Avron, Y., Zak, J.: Quantised Hall conductance in a perfect crystal. J. Phys. C 18, L679 (1985)

6. Hatsugai, Y.: Chern number and edge states in the integer quantum Hall effect. Phys. Rev. Lett. 71, 3697-3700 (1993)

7. Hatsugai, Y., Fukui, T., Aoki, H.: Topological analysis of the quantum Hall effect in graphene: DiracFermi transition across van Hove singularities and edge versus bulk quantum numbers. Phys. Rev. B 74, 205414 (2006)

8. Hofstadter, D.R.: Energy levels and wave functions of Bloch electrons in rational and irrational magnetic fields. Phys. Rev. B 14, 2239-2249 (1976)

9. Kreft, C., Seiler, R.: Models of the Hofstadter-type. J. Math. Phys. 37, 5207-5243 (1996)

10. MacDonald, A.H.: Quantized Hall effect in a hexagonal periodic potential. Phys. Rev. B 29, 3057-3065 (1984)

11. Porta, M., Graf, G.M.: Bulk-edge correspondence for two-dimensional topological insulators. Commun. Math. Phys. 324, 851-895 (2013)

12. Rammal, R.: Landau level spectrum of Bloch electrons in a honeycomb lattice. J. Phys 46, 1345-1354 (1985)

13. Sato, M., Tobe, D., Kohmoto, M.: Hall conductance, topological quantum phase transition, and the Diophantine equation on the honeycomb lattice. Phys. Rev. B 78, 235322 (2008)

14. Streda, P.: Theory of quantised Hall conductivity in two dimensions. J. Phys. C 15, L717-L721 (1982)

15. Thouless, D.J., Kohmoto, M., Nightingale, M.P., den Nijs, M.: Quantized Hall conductance in a twodimensional periodic potential. Phys. Rev. Lett. 49, 405-408 (1982) 